\title{
Granulomatous Mastitis Complicated by Arthralgia and Erythema Nodosum Successfully Treated with Prednisolone and Methotrexate
}

\author{
Tomoyuki Nakamura ${ }^{1}$, Katsunobu Yoshioka ${ }^{2}$, Tomoko Miyashita ${ }^{1}$, Katsumi Ikeda ${ }^{3}$, \\ Yoshinari Ogawa ${ }^{3}$, Takeshi Inoue ${ }^{4}$ and Keiko Yamagami ${ }^{1}$
}

\begin{abstract}
A 23-year-old woman was admitted with complaints of swelling and pain in the left breast, fever, polyarthralgia and erythema nodosum. A fine-needle biopsy of the mass in the left breast revealed non-caseous granulomatous lobulitis. A diagnosis of granulomatous mastitis was thus made. The administration of prednisolone $40 \mathrm{mg} /$ day resulted in the resolution of the patient's symptoms, and the breast mass thereafter decreased in size. The mass relapsed during the subsequent prednisolone taper. Additional therapy with methotrexate resulted in complete remission. Granulomatous mastitis should therefore be included in the differential diagnosis of polyarthralgia.
\end{abstract}

Key words: granulomatous mastitis, erythema nodosum, polyarthralgia, glucocorticoids, methotrexate

(Intern Med 51: 2957-2960, 2012)

(DOI: 10.2169/internalmedicine.51.7846)

\section{Introduction}

Granulomatous mastitis is a rare, benign and chronic inflammatory breast disease first described by Kessler and Wolloch in 1972 (1). It is clinically characterized by hard breast lumps with local inflammation and histologically by the presence of non-caseous granulomatous lobulitis. Polyarthritis and erythema nodosum, as opposed to fever, are rare systemic manifestations of granulomatous mastitis. As far as we know, only three such cases have so far been reported (2-4).

The etiology of granulomatous mastitis remains unknown. However, the histological findings and responsiveness to systemic glucocorticoid therapy suggest that the disease is caused by immune-mediated mechanisms.

We herein report the case of a patient with granulomatous mastitis complicated by severe polyarthralgia and erythema nodosum who was successfully treated with systemic glucocorticoid and methotrexate (MTX) therapy. Additionally, the rationale for using such treatment strategies is discussed.

\section{Case Report}

A 23-year-old woman was admitted to our hospital in August of 2009 complaining of swelling and pain in the left breast, fever, an erythematous lesion on her leg and severe polyarthralgia. She had been in good health until one month prior when she noticed swelling and pain in her left breast. She had no history of parturition, galactorrhea or breast injury and was taking no medications. She presented with a high fever and severe symmetrical polyarthralgia and was valuated at the breast surgery department of our hospital. Due to the fact that she had chills and could not move as a result of the severe polyarthralgia, she was referred to our department and admitted to the hospital.

On admission, the patient was $163 \mathrm{~cm}$ in height and weighed $60 \mathrm{~kg}$. Her temperature was $38^{\circ} \mathrm{C}$, her pulse was 76 beats/min and her blood pressure was $100 / 60 \mathrm{mmHg}$. On physical examination, pain in the entire left breast accompa-

\footnotetext{
${ }^{1}$ Department of Internal Medicine, Osaka City General Hospital, Japan, ${ }^{2}$ Department of Internal Medicine, Osaka City Sumiyoshi Hospital, Japan, ${ }^{3}$ Department of Breast Surgical Oncology, Osaka City General Hospital, Japan and ${ }^{4}$ Department of Pathology, Osaka City General Hospital, Japan

Received for publication March 28, 2012; Accepted for publication July 11, 2012

Correspondence to Dr. Tomoyuki Nakamurai, tomosquared@yahoo.ne.jp
} 
Table. Laboratory Data on Admission

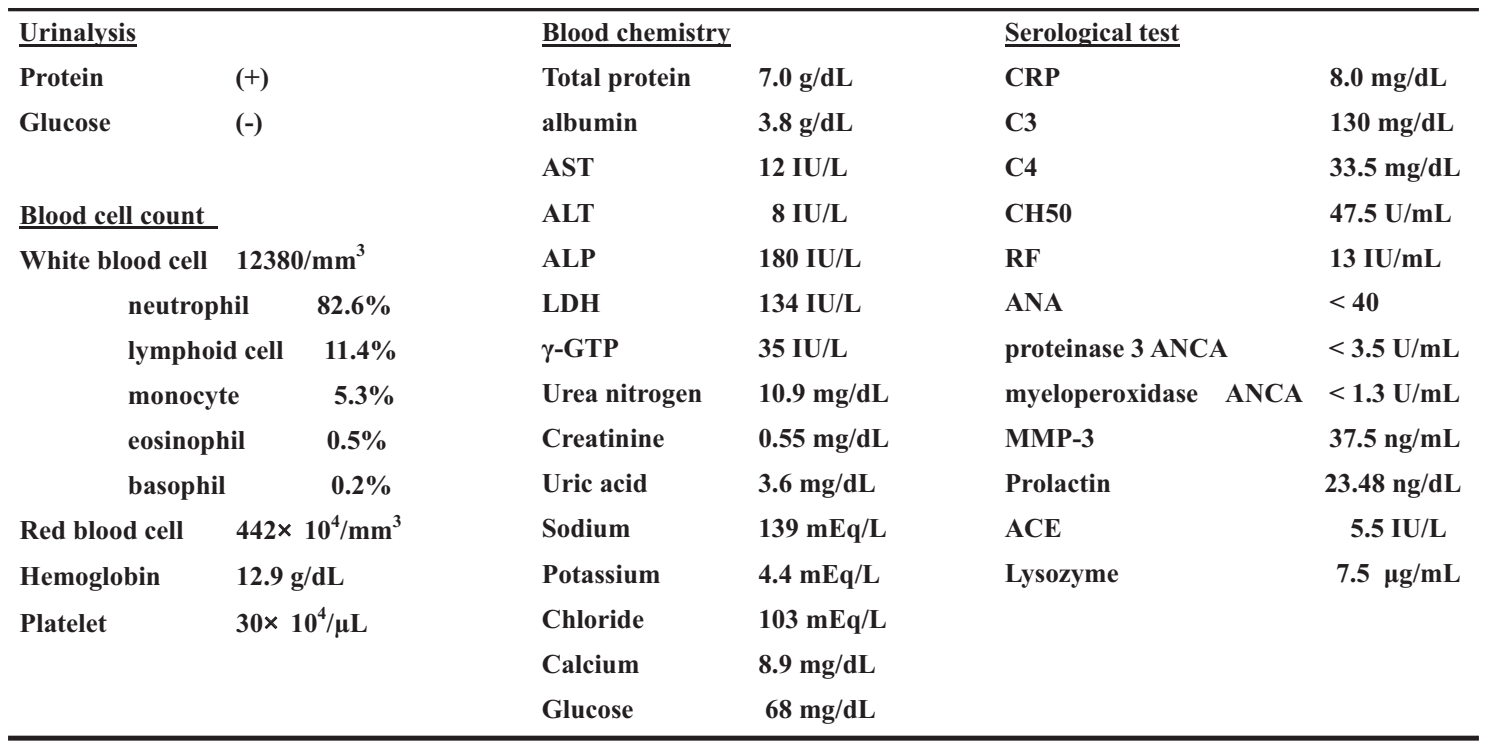

AST: aspartate aminotransferase, ALT: alanine aminotransferase, LDH: lactate dehydrogenase, $\gamma$-GTP: $\gamma$-glutamyl transpeptidase, ALP: alkaline phosphatase, CRP: C-reactive protein, RF: rheumatoid factor, ANA: anti-nuclear antibody ANCA: antineutrophil cytoplasmic antibody, MMP-3: Matrix Metalloproteinase-3,ACE: angiotensin conveting enzyme

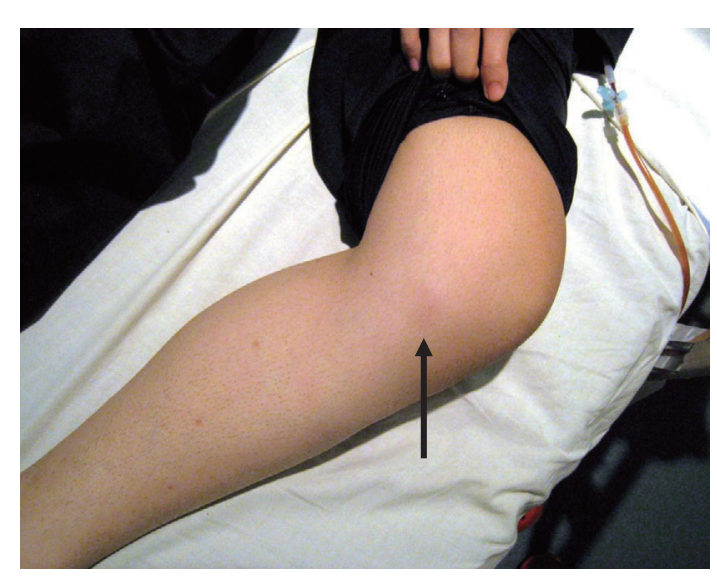

Figure 1. Pretreatment clinical findings showing erythema nodosum with pain in the lower extremities.

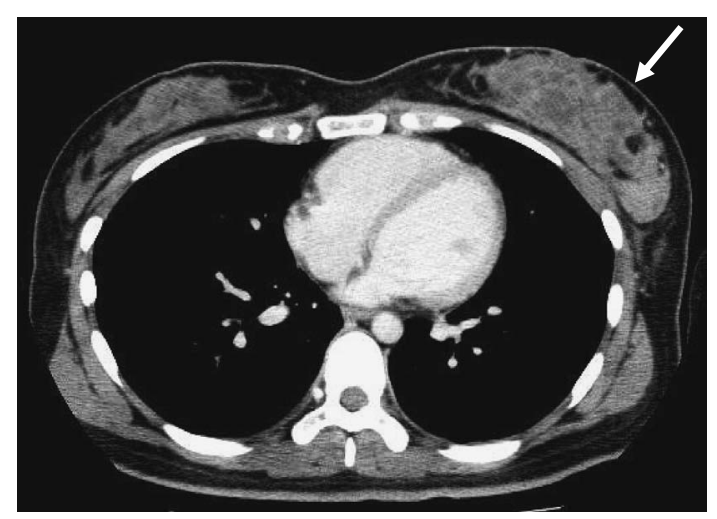

Figure 3. Pretreatment contrast-enhanced computed tomography $(\mathrm{CT})$ showing a mass lesion with ring enhancement in the left breast.

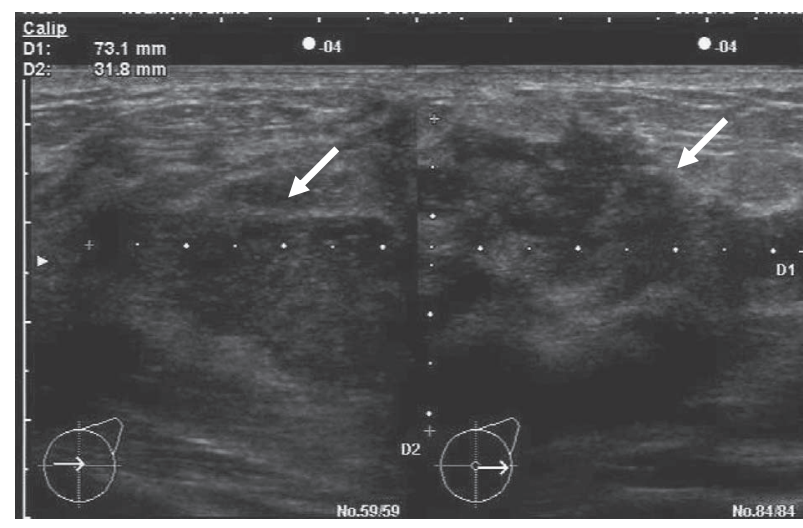

Figure 2. Ultrasound demonstrating a $7.3 \times 3.2 \times 6.7 \mathrm{~cm}$ hypoechoic mass lesion consisting of many small lesions and fluid in the left breast.

nied by a mass was observed, as well as erythema nodosum of the left lower extremity (Fig. 1). The remainder of the physical examination was unremarkable. Laboratory tests on admission revealed elevated C-reactive protein levels and leukocytosis (Table). Ultrasonography revealed multiple abscesses in the left breast (Fig. 2). Computed tomography (CT) revealed multiple abscesses in the left breast and left axillary lymph node (Fig. 3). A diagnosis of arthritis and erythema nodosum caused by bacterial infection was initially suspected. After draining the contents of the abscess, the patient was started on antibiotic therapy with cefazolin. However, the symptoms and inflammation did not improve. Bacterial cultures of the blood and aspiration specimens were negative. Because a diagnosis of granulomatous mastitis was suspected at this point, a fine-needle biopsy of the mass was performed. Microscopic features showed a noncaseating granuloma composed of epithelioid cells. Multinu- 


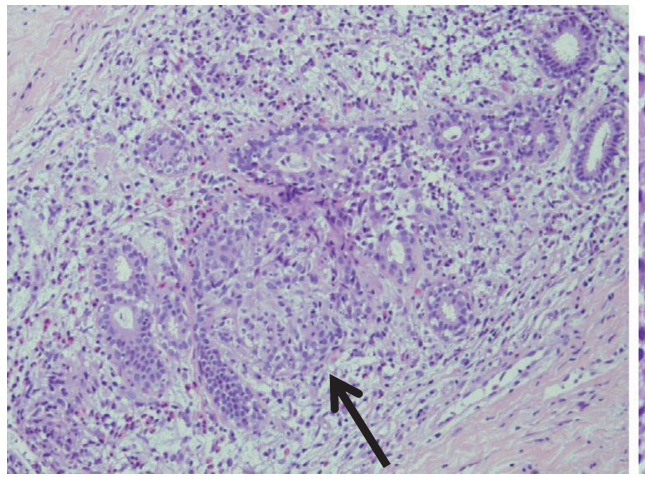

A

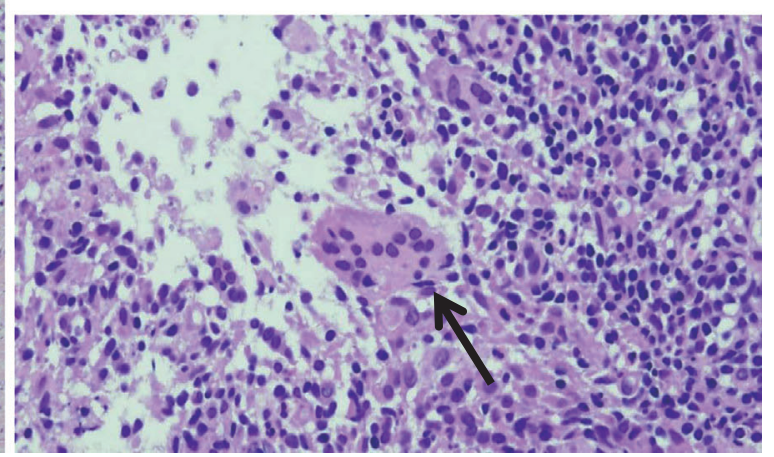

B

Figure 4. Histopathological examination with Hematoxylin and Eosin staining showing a noncaseating granuloma composed of epithelioid cells $(\mathrm{A})(\times 400)$. Multinucleated giant cells were also present (B).

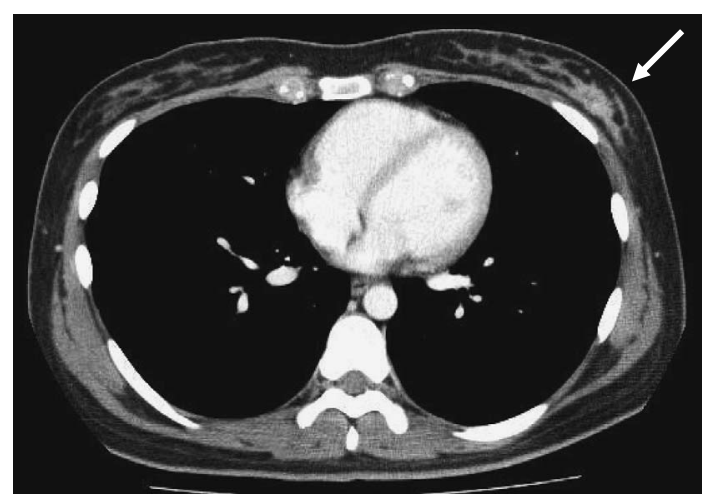

Figure 5. Post-treatment enhanced CT showing the mass lesion reduced in size.

cleated giant cells were also present; however, no microorganisms were detected (Fig. 4). A diagnosis of sarcoidosis and Wegener's granulomatosis was thus suspected. However, a diagnosis of sarcoidosis was thought to be unlikely because both angiotensin converting enzyme and the lysozyme levels were normal, and also because the granulomatous lesion was confined to the breast. Wegener's granulomatosis was ruled out because tests for auto-antibodies, including proteinase 3 ANCA, were negative. After tuberculosis was also ruled out by normal chest X-rays and histological findings, a diagnosis of granulomatous mastitis was thus confirmed.

Since this patient had arthralgia and systemic inflammation, prednisolone $40 \mathrm{mg} /$ day was administered starting on the 8th day of hospitalization according to the standard medical treatment of systemic granulomatous disease. The following day, this treatment regimen led to the resolution of the fever, erythema nodosum and arthralgia, and the patient regained the ability to walk independently. During the second week of treatment, the pain in the patient's left breast improved and the CRP level normalized. Prednisolone was tapered by $5 \mathrm{mg}$ every two weeks, and the abscess in the left breast became smaller on CT (Fig. 5). Two months later, while the patient was taking $15 \mathrm{mg} /$ day of predniso- lone, the swelling and pain in the left breast recurred. The dose of prednisolone was then increased to $50 \mathrm{mg} /$ day; however, the symptoms did not improve. Surgery was contraindicated due to fistula formation. MTX $4 \mathrm{mg} / \mathrm{week}$ was added based on reports that it may be effective in patients that show a lack of response to steroids (5). One month after adding MTX, the patient's symptoms improved. The dose of prednisolone was gradually tapered to $5 \mathrm{mg} / \mathrm{day}$ over eight months, and $4 \mathrm{mg} /$ week of MTX was continued for one year. During the 5-month course of prednisolone administration, no disease recurrence was observed. Thereafter, the prednisolone dose was tapered to $1.25 \mathrm{mg} /$ day over three months. Two years later, the abscess was found to have disappeared and MTX was therefore discontinued. The patient is now being treated with prednisolone $1.25 \mathrm{mg} /$ day and has not experienced any further recurrence of the disease.

\section{Discussion}

Granulomatous mastitis usually presents with a unilateral breast lump, breast pain, nipple inversion and axillary lymphadenopathy. Because these symptoms are also seen in breast cancer and because granulomatous mastitis has no specific radiological appearance (6), it is difficult to clinically distinguish granulomatous mastitis from breast cancer. A histological examination is therefore essential for a proper diagnosis (7). The characteristic histological findings are lobular non-caseous granulomas with epithelioid histiocytes, giant cells and neutrophils and a negative microbiological investigation.

Due to histological similarities between the diseases, the differential diagnosis of granulomatous mastitis also includes tuberculosis, sarcoidosis, Wegener's granulomatosis, fungal infection and aseptic abscesses syndrome (8). Aseptic abscesses syndrome, a clinicopathological entity first described in 1995, is characterized by recurrent manifestations of abscess-like areas that respond to corticosteroid therapy but not to antibiotics. Patients with aseptic abscesses syndrome also present with arthritis as well as pyoderma gangreno- 
sum. Histologically, the abscess-like lesions contain mature neutrophils, epithelioid cells and giant cells (9). Therefore, clinical and histological similarities exist between granulomatous mastitis and aseptic abscesses syndrome. However, the typical presentation of patients with aseptic abscesses syndrome is characterized by the presence of aseptic lesions that are usually located in the spleen, liver and abdominal lymph nodes. In contrast, the abscess in the present case was localized to the breast and the skin lesion was typical of erythema nodosum. Therefore, the possibility of aseptic abscesses syndrome was considered to be low. It is necessary to distinguish these diseases based on both histological and clinical findings. The present case was diagnosed with granulomatous mastitis based on both findings, as described above.

Polyarthritis and erythema nodosum, as opposed to fever, are rare systemic manifestations of granulomatous mastitis. In 1987, Adams et al. reported the first case of granulomatous mastitis complicated by arthritis and erythema nodusum (2). Although cases of granulomatous mastitis complicated solely by erythema nodusum are occasionally reported, as far as we know, only two other cases of granulomatous mastitis complicated by both arthritis and erythema nodusum have been previously reported $(3,4)$. Because granulomatous mastitis usually develops in association with lactation, hyperprolactinemia or breast injury, an immune response to extravasated secretions from lobules has been proposed as an underlying mechanism. However, the present patient had no history of parturition, galactorrhea or breast injury. Therefore, this mechanism was unlikely. The presence of a microabscess implies an infective cause of granulomatous mastitis. However, no infectious agents were identified, despite extensive investigation. Therefore, the etiology of granulomatous mastitis in this case is unknown. However, the complications of polyarthritis and erythema nodosum and the responsiveness to glucocorticoid therapy suggest that the granulomatous mastitis in this case was caused by immune-mediated mechanisms. However, both auto-antibodies and auto-antigen-specific $\mathrm{T}$ cells are absent in patients with granulomatous mastitis. Therefore, granulomatous mastitis may be a form of autoinflammatory disease.

No consensus exists concerning the optimal treatment modality for patients with granulomatous mastitis. The treatment used in the present report consisted of corticosteroids and surgical excision. Patients without symptoms may be observed without treatment. It has been reported that $50 \%$ of granulomatous mastitis cases resolve spontaneously without treatment and a complete resolution occurs at a mean of 14.5 months (range: 2-24 months) (10). It was reported in 1980 that treatment with systemic corticosteroids is an effective therapy for granulomatous mastitis (11). However, an optimal dose of corticosteroid therapy has not yet been established, and the disease can recur when corticosteroids are tapered. Considering that the present case was complicated by severe arthralgia and erythema nodosum and an immunemediated mechanism was suspected, corticosteroids were administered. In the present case, the initial response to prednisolone was excellent. However, the granulomatous mastitis relapsed when the dose of prednisolone was tapered to 15 $\mathrm{mg} /$ day. It has been reported that a low weekly dose of MTX may be effective in cases with resistance to corticosteroids $(5,12)$. Therefore, MTX was added to the patient's therapy. Thereafter, the dose of prednisolone was reduced, leading to complete remission. The patient's symptoms did not improve with increases in prednisolone; however, they did improve with the addition of low-dose MTX. The success of the clinical course of the present case was thought to be attributable to the efficiency of MTX plus prednisolone, not to the natural course of the disease.

In summary, we herein reported a rare case of granulomatous mastitis complicated by symmetrical arthralgia and erythema nodosum. MTX was used to reduce the dose of prednisolone, which resulted in complete remission. Physicians should therefore be aware of the existence of granulomatous mastitis in order to properly treat patients presenting with polyarthralgia.

\section{The authors state that they have no Conflict of Interest (COI).}

\section{References}

1. Kessler E, Wolloch Y. Granulomatous mastitis: a lesion clinically simulating carcinoma. Am J Clin Pathol 58: 642-646, 1972.

2. Adams DH, Hubscher SG, Scott DG. Granulomatous mastitis: a rare cause of erythema nodosum. Postgrad Med J 63: 581-582, 1987.

3. Olfatbakhsh A, Beheshtian T, Djavid GE. Granulomatous mastitis, erythema nodosum, and oligoarthritis in a pregnant woman. Breast J 14: 588-590, 2008.

4. Salesi M, Karimifar M, Salimi F, Mahzouni P. A case of granulomatous mastitis with erythema nodosum and arthritis. Rheumatol Int 31: 1093-1095, 2011.

5. Kim J, Tymms KE, Buckingham JM. Methotrexate in the management of granulomatous mastitis. ANZ J Surg 73: 247-249, 2003.

6. Lee JH, Oh KK, Kim EK, Kwack KS, Jung WH, Lee HK. Radiologic and clinical features of idiopathic granulomatous lobular mastitis mimicking advanced breast cancer. Yonsei Med J 47: 7884, 2006.

7. Martinez-Parra D, Nevado-Santos M, Melendez-Guerrero B, Garcia-Solano J, Hierro-Guilmain CC, Perez-Guillermo M. Utility of fine-needle aspiration in the diagnosis of granulomatous lesions of the breast. Diagn Cytopathol 17: 108-114, 1997.

8. Baslaim MM, Khayat HA, Al-Amoudi SA. Idiopathic granulomatous mastitis: a heterogeneous disease with variable clinical presentation. World J Surg 31: 1677-1681, 2007.

9. Andre M, Aumaitre O, Marcheix JC, Piette JC. Aseptic systemic abscesses preceding diagnosis of Crohn's disease by three years. Dig Dis Sci 40: 525-527, 1995.

10. Imoto $S$, Kitaya $T$, Kodama $T$, Hasebe $T$, Mukai K. Idiopathic granulomatous mastitis: case report and review of the literature. Jpn J Clin Oncol 27: 274-277, 1997.

11. Lai EC, Chan WC, Ma TK, Tang AP, Poon CS, Leong HT. The role of conservative treatment in idiopathic granulomatous mastitis. Breast J 11: 454-456, 2005.

12. Akbulut S, Yilmaz D, Bakir S. Methotrexate in the management of idiopathic granulomatous mastitis: review of 108 published cases and report of four cases. Breast J 17: 661-668, 2011.

(C) 2012 The Japanese Society of Internal Medicine http://www.naika.or.jp/imonline/index.html 